\title{
A RFID Anti-collision Algorithm based on Q-ary Search
}

\author{
Xinyan Wang, Lijun Mao \\ School of Intelligence Science and Information Engineering \\ Xi'an Peihua University, Xi'an, China \\ 36404843@qq.com,35909250@qq.com
}

Key words: RFID; Anti-collision; Multiple tags identification; QAS

\begin{abstract}
Multiple tags collision problem severely degrade the identification efficiency of an RFID system. As a mainstream RFID anti-collision algorithm, deterministic tree-based algorithm can guarantee that the reader correctly identify the tags within its coverage range. In order to overcome the shortcomings of the existing tree-based algorithms, a Q-ary search (QAS) algorithm with tag ID bit encoding mechanism is proposed. The QAS algorithm introduced bit encoding mechanism of tags' ID by which the multi-bit collision arbitration can be implemented by the reader. Benefiting from the encoding mechanism, the collision slots is reduced, and the identification efficiency is improved. The theoretical analysis and simulation results show that the QAS algorithm reduces the time complexity and communication overhead during the identification process and achieves the good performance compare to the references methods.
\end{abstract}

\section{Introduction}

As the most promising automatic identification technology, radio frequency identification (RFID) is playing an important role in many areas [1], such as access control, supply chain management, transportation and so on. Compared with traditional barcode, RFID has many advantages, such as fast scan, compact size, anti-pollution ability, reusable, good penetrability and large data storage capacity. Therefore, it has become one of the core technologies of the Internet of things [2]. In RFID technology, there are multiple tag collision problems, that is, when the reader communicates with multiple tags at the same time, the signal of the tag may collide, which makes the reader unable to identify the tag correctly. Therefore, the corresponding collision avoidance algorithm is needed to solve the problem of tag collision.

The mainstream anti-collision algorithms can be divided into two categories: the ALOHA algorithm and the tree algorithm. The basic principle of ALOHA algorithm is in the process of identifying, reader response detection tag and determine whether the collision, if the collision, the reader sends commands to stop sending data and tag, tag each random delay for a period of time and then sent to the reader data, this mechanism is also called random concession mechanism. According to the difference of the concession mechanism, the ALOHA algorithm can also be divided into the slot ALOHA (SA) algorithm, the framed slotted ALOHA (FSA) algorithm and the dynamic framed slotted ALOHA (DFSA) algorithm[3-5]. The Alaha algorithm is based on the time slot to identify the tags. The algorithm is simple and has good performance when the number of tags is less. However, due to the random generation of time slots, there is a certain possibility that a certain tag cannot be identified for a long time, that is, the problem of tag hunger [5]. At the same time, the energy of the passive tag and the frame length specified by the reader are limited. With the increase of the number of tags, the recognition performance of the algorithm will decline sharply. 
The tree algorithm can be roughly divided into query tree algorithm (QT) [6-7], binary search algorithm (BS) and tree separation algorithm (TS) [8-10]. Compared with the ALOHA algorithm, the tree algorithm does not have the "tag hunger" problem, but when the tag recognition code is long, there will be a large number of collision slots.

In this paper, in view of the shortcomings of the existing tree-based algorithm, a M-ary anti-collision algorithm is proposed by introducing the bit encoding mechanism of tags' ID. Through the introduction of bit encoding mechanism of tags' ID, the algorithm can achieve multi-bit collision arbitration, which greatly reduces the number of collision slots in the recognition process, and effectively improves the recognition efficiency of RFID system.

\section{The Preliminaries}

In traditional tree-based algorithm, no matter whether there is collision in the tree node, the algorithm protocol will traverse the entire binary tree, which results in the low efficiency of the traditional tree-based algorithm. To this end, researchers have proposed a series of improved tree-based algorithms.

A collision tree algorithm (CT) is proposed [11], which can effectively eliminate idle time slot generated by reader query process, thereby improving the performance of binary search algorithm, and the system throughput is maintained at around 50\%. In another paper, an identification anti-collision algorithm (IACA) based on continuous collision location recognition is proposed in the literature [12]. By detecting whether there is continuous collision in high position, the algorithm can assign four, 00, 01, 10 and 11 of tag collision information separately. The algorithm effectively reduces the number of collisions when the tag is large, thus improving the efficiency of system recognition. However, if the continuous collision bit is caused by less tag collisions, the system produces too many idle time slots, resulting in a decline in performance. Based on adaptive M-ary search algorithm (AMS) [13], an improved adaptive M-ary search algorithm (IAMS) is proposed by optimizing the query prefix of reader. The algorithm eliminates idle time slot in AMS algorithm, and further improves the efficiency of system recognition. The system throughput can be increased to about $60 \%$. However, due to the AMS algorithm and IAMS algorithm with M-ary splitting is based on collision factor depends on the value of the number of tags collision factor and actual reaction cannot accurately, so a lot of time, this algorithm is not as expected by four hexadecimal split to reduce collision slots, which leads to the decline in performance.

In the algorithm, this paper proposes a multi tag ID bit encoding mechanism based on Q-ary search algorithm (QAS), the algorithm through the ID tag encoding mechanism can effectively eliminate idle time, reduce the number of collision, so as to improve the recognition efficiency of the RFID system.

\section{QAS Algorithm}

In the QAS algorithm, we use the bit coding mechanism to implement multi bit arbitration, thus improving the speed of the reader and reducing the number of collisions.

The data before the code is a $\mathrm{k}$ bit data string $\mathrm{S}=\mathrm{bk}-1 \mathrm{bk}-2 \ldots \mathrm{b} 0$, where bi represents binary values 0 or 1 . The encoded data is a data string with a length of $Q=2 k$ bits: $S 1=d Q-1 d Q-2 . . d 0$, where di represents binary values, and in the data string $\mathrm{Q}$ bits have 1 bit for 1 , and the rest are 0 .

The encoded data contain only one 1, the advantage of this is that, when the reader receives the data after encoding can detect collision information tags accurately, without additional custom 
commands and the introduction of idle time, it will to further improve the efficiency of system identification.

Considering the difference of data transmission in single time slot in different algorithms, we analyze the performance of QAS algorithm through three indexes, such as time slot number, throughput and energy efficiency. It is assumed that there are $n$ ID lengths of $m=8$ to be identified in the system. A standard equivalent slots includes a query command segment and a tag complete ID data, then the equivalent time slots required by the algorithm can be calculated as:

$$
T_{\text {equ }}=\frac{\sum_{i=0}^{T_{\text {total }}} L_{i}}{L_{c m d}+m}
$$

Among them, $\mathrm{T}_{\text {total }}$ is the number of query cycles needed for the whole recognition process, $\mathrm{Li}$ is the amount of data transmitted by I query, and $\mathrm{L}_{\mathrm{cmd}}$ is the amount of transmission data occupied by query command. When the initialization query is considered, the continuous collision bit length of the tag is $\mathrm{k}$, and the search depth required for the entire identification process is $1+1+\left\lceil\frac{m-k}{2}\right\rceil$. It can be analyzed that the entire query tree contains 1 root nodes, $2^{k+\left\lceil\frac{m-k}{2}\right]^{-1}}$ intermediate nodes and $2^{k+\left\lceil\frac{m-k}{2}\right\rceil}$ leaf nodes, So $\mathrm{T}_{\text {total }}$ can be calculated as:

$$
T_{\text {total }}=\left\{\begin{array}{l}
\sum_{j=0}^{1+1+\left\lceil\frac{m-k}{2}\right\rceil} N_{j} P_{c o l}^{j}+n, n \geq 1 \\
1, \quad n=1
\end{array}\right.
$$

$\mathrm{Nj}$ represents the number of nodes that are included in the search depth of $\mathrm{J},{ }^{P_{c o l}^{j}}$ represents the probability that a single node has a collision in the search depth of $\mathrm{J}$. The root node for $\mathrm{j}=0$ is always able to be queried. For other nodes outside the root node, the premise that can be queried is that its parent node produces collisions. We define the probability that $\mathrm{P}(\mathrm{i}, \mathrm{j})$ has I sub nodes in a single node in the search depth $\mathrm{j}$, then we can get:

$$
\begin{aligned}
& P_{c o l}^{j}= \begin{cases}1, & j=0,1 \\
\sum_{j=2}^{1+1+\left\lceil\frac{m-k}{2}\right\rceil} \sum_{i=2}^{N_{j}} P(i, j) & 2 \leq j \leq 2+\left\lceil\frac{m-k}{2}\right\rceil\end{cases} \\
& P(i, j)=C_{n}^{i}\left(\frac{C_{2^{m-k} / 2^{j-2}}^{i}}{C_{2^{m}}^{i}}\right)\left(\frac{C_{2^{m}-2^{m-k} / 2^{j-2}}^{n-i}}{C_{2^{m}-i}^{n-i}}\right)
\end{aligned}
$$

Through the above analysis, we conclude that the throughput rate of the system is:

$$
u=\frac{n}{T_{\text {equ }}}
$$


In order to better evaluate the recognition performance of the algorithm, we define the energy efficiency index to measure the energy consumption of the algorithm:

$$
\eta=\frac{n}{Q . e_{q}+S \cdot e_{s}+R \cdot e_{r}} e_{u}
$$

$\mathrm{Q}$ is the number of queries for readers, $\mathrm{S}$ is the number of successful slots, and $\mathrm{R}$ is the total number of tag responses. $\mathrm{e}_{\mathrm{q}}, \mathrm{e}_{\mathrm{s}}$ and $\mathrm{e}_{\mathrm{r}}$ correspond to the corresponding energy consumption, respectively, and eu is the unit energy consumption.

\section{Simulation Analysis}

We verified the performance of QAS algorithm through experimental simulation, taking into account 3 indexes: the number of equivalent slots, the average recognition cycle and energy efficiency. In simulation, we randomly generate 5-500 tags with ID length of 16bit, and simulate the recognition process of CT algorithm, IAMS algorithm, IAC algorithm and QAS algorithm based on the above 3 indexes.

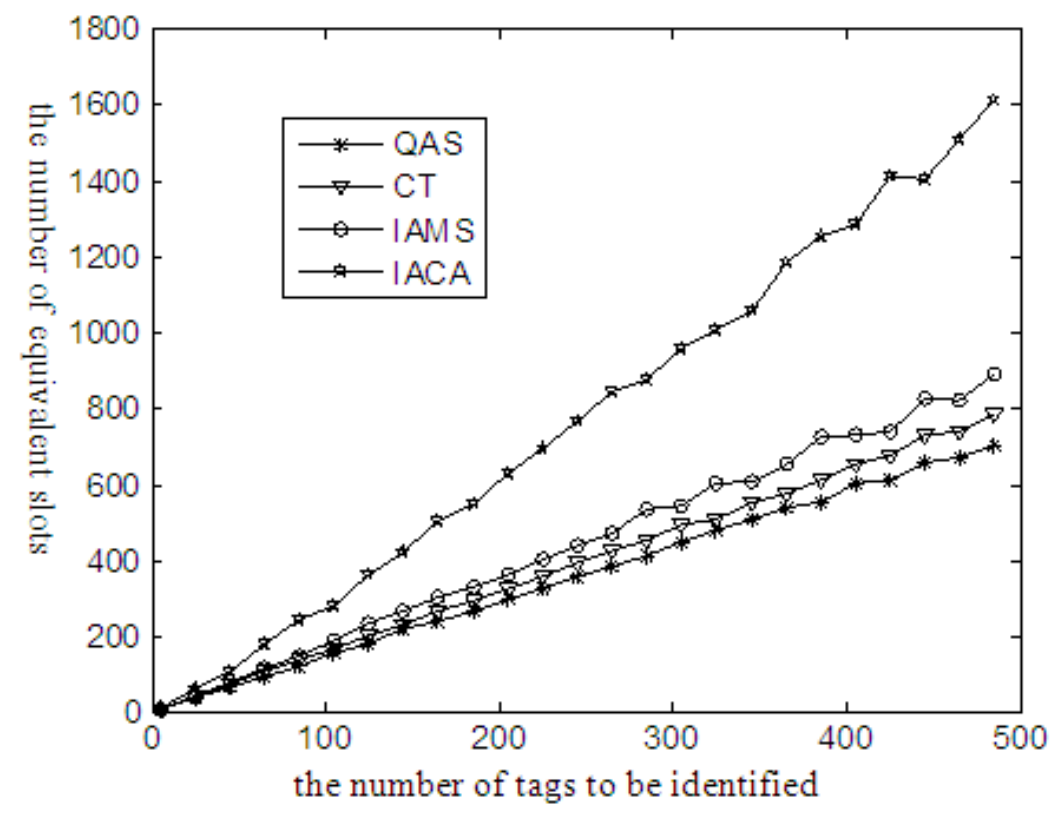

Figure 1 Comparison of the equivalent time slot number required by each algorithm to identify the same number of tags

Figure 1 compares the CT algorithm, the IAMS algorithm, the IAC algorithm and the equivalent time slot number of the QAS algorithm. It can be seen that the QAS algorithm is obviously superior to other algorithms. Especially when the number of tags exceeds 200, the performance advantage of QAS algorithm is more obvious. For example, when the number of tags is 305, the time slot numbers of IACA, CT and IAMS algorithms are 957, 492 and 545, respectively. At the same time, QAS only needs 448 slot to identify 305 tags. 


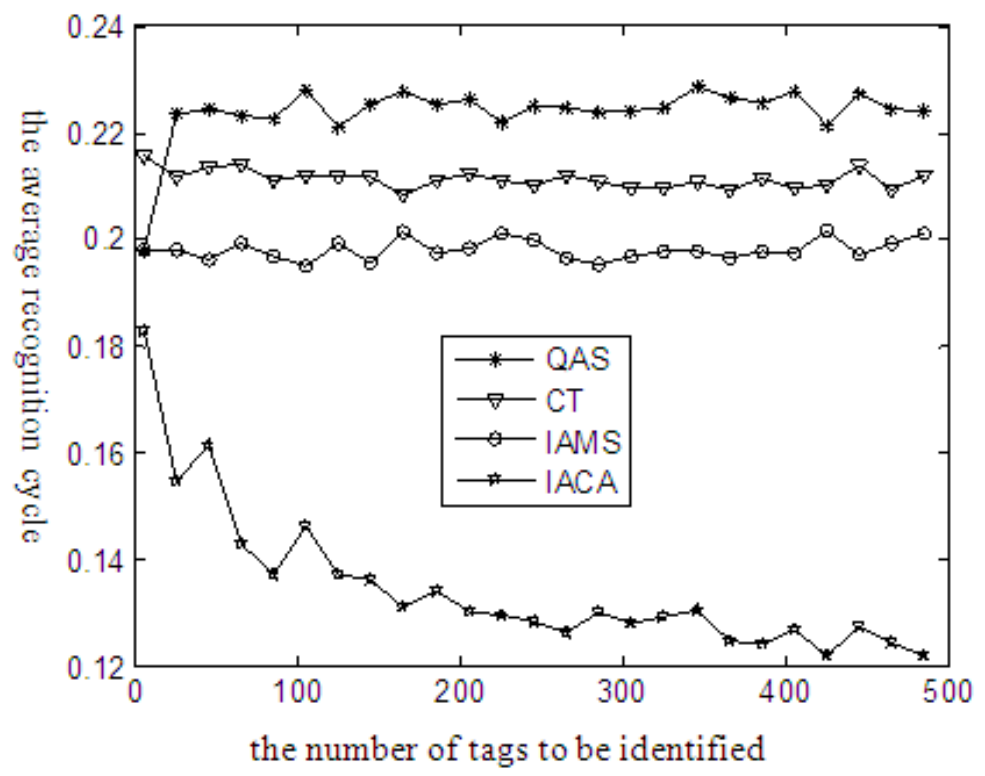

Figure 2 Comparison of average recognition cycle

Figure 2 shows the average number of query cycles required for a different algorithm to complete a single tag recognition, and the total number of tags varies between 5-500. It can be seen from the graph that the QAS algorithm for identifying single tags needs only 1.5 rounds of query cycles on average, while IACA, CT and IAMS require 3,1.8 and 1.65 rounds of query cycles respectively.

Although the QAS algorithm uses the encoded data of tag ID, and the data of tag response is divided into 2 parts, it has little influence on the communication complexity of the whole system. As you can see from Figure 3, the QAS algorithm has the highest energy efficiency, in other words, identifying the same number of tags, the QAS algorithm will consume less energy. The energy efficiency of the QAS algorithm remains above 0.22 , which is higher than the IACA, CT, and IAMS algorithms, $65 \%, 6 \%$ and $14 \%$.

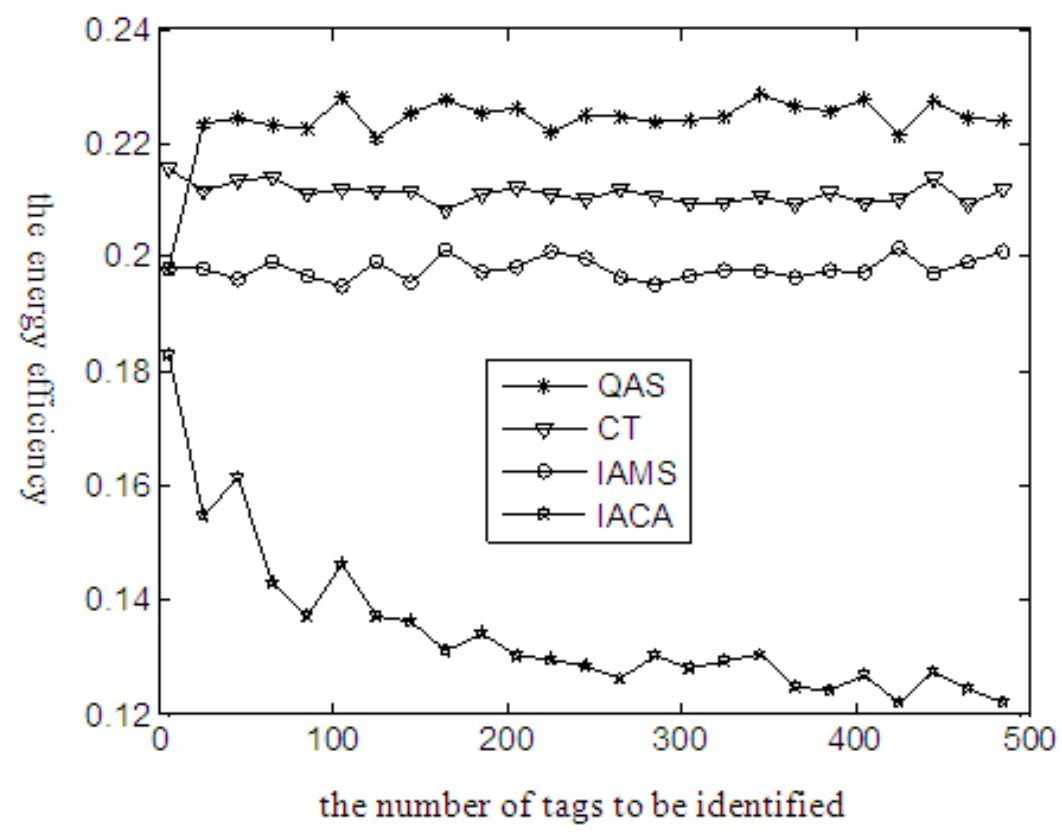

Figure 3 Comparison of energy efficiency 


\section{Conclusion}

In this paper, a Q-ary search (QAS) algorithm is proposed.. In the QAS algorithm, the tag ID encoding mechanism is introduced. Through this mechanism, the reader can implement multi bit collision arbitration for tags, avoiding the generation of idle time slot and reducing the number of collision slots, thus improving the recognition efficiency of the system. At the same time, in the design of the algorithm, the problem of information transmission and energy consumption is fully considered. The performance of the algorithm is evaluated by the three indexes of the equivalent slot number, the number of average query cycles and the energy efficiency. The theoretical analysis and simulation results show that QAS algorithm reduces the time complexity and communication overhead during the identification process, and has good performance under the condition of large number of tags.

\section{Reference}

[1] Wang Y, Liu Y, Leung H, etal. 2013. A multi-bit identification protocol for RFID tag reading, IEEE Sensor Journal, 13(10): 3527-3536.

[2] Ning S, Sha H, Wei H, etal. 2012. ID-based Internet of Things and its application in airport aviation risk management, Chinese Journal of Electronics, 21(2): 209-214.

[3] Zhang Xiaohong, Hu Yingmeng. 2016. Research on RFID anti-collision algorithm for packet adaptive allocation of time slot, Electronic journal, 44(6): 1328-1335.

[4] Myung J, Lee W, and Shih T K. 2006. An adaptive memory less protocol for RFID tag collision arbitration, IEEE Transactions on Multimedia, 8(5): 1096-1101..

[5] Suzhou, Han Yu, Luo Zhongqiang. 2015. A feasible time optimal anti-collision algorithm in UHF RFID system, Electronic journal, 43(8): 1651-1655.

[6] Kim Y, Kim S, Lee S, etal. 2009. Improved 4-ary query tree algorithm for anti-collision in RFID system, Proceedings of International Conference on Advanced Information Networking and Applications, Bradford, United Kingdom, 699-704.

[7] Wang Xin, Jia Qingxuan, Gao Xin. 2016. Research on anti-collision algorithm of block N fork tracking tree RFID, Electronic journal, 44(2): 437-444.

[8] Myung J, Lee W, and Srivastava J. 2006. Adaptive binary splitting for efficient RFID tag anti-collision, IEEE Communications Letters, 8(5): 1096-1101.

[9] Cui Y and Zhao Y. 2010. Performance evaluation of a multi-branch tree algorithm in RFID, IEEE Transactions on Communications, 58(5): 1356-1364.

[10] Su J, Sheng Z, Xie L, and Wen G. 2016. Idle-slots elimination based binary splitting anti-collision algorithm for RFID, IEEE Communications Letters, 20(12): 2394-2397.

[11]Jia X, Feng Q, and Ma C. 2010. An efficient anti-collision protocol for RFID tag identification, IEEE Communications Letters, 14(11): 1014-1016.

[12]Djeddou M, Khelladi R, and Benssalah M. 2013. Improved RFID anti-collision algorithm, AEU-International Journal of Electronics and Communications, 67(3): 256-262.

[13]Zhang Xuejun, Cai Wenqi, Wang Suoping. 2012. Study on improved adaptive multi fork collision avoidance algorithm, Electronic journal, 40(1): 193-198. 